\title{
Decentralized Control For Guaranteed Individual Costs in a Linear Multi-agent System: a Satisfaction Equilibrium Approach
}

\author{
J. Veetaseveera, V.S. Varma, I.C. Morărescu and J. Daafouz
}

\begin{abstract}
This work focuses on the design of decentralized feedback control gains that aims at optimizing individual costs in a multi-agent synchronization problem. As reported in the literature, the optimal control design for synchronization of agents using local information is NP-hard. Consequently, we relax the problem and use the notion of satisfaction equilibrium from game theory to ensure that each individual cost is guaranteed to be lower than a given threshold. Our main results provide conditions in the form of linear matrix inequalities (LMIs) to check if a given set of control gains are in satisfaction equilibrium i.e. all individual costs are upper-bounded by the imposed threshold. Moreover, we provide an algorithm in order to synthesize gains that are in satisfaction equilibrium. Finally, we illustrate this algorithm with numerical examples.
\end{abstract}

Index Terms-Control of networks, Decentralized control

\section{INTRODUCTION}

D ECENTRALIZED coordination of multi-agent systems has attracted a lot of attention during the past decade. In the decentralized control design paradigm, each system is able to design and implement its own control law without the help of a central entity. The main goal of this paper is to design a control strategy that can be implemented in a decentralized manner and allows multi-agent systems to achieve synchronization with local or individual performance guarantees.

The multi-agent formalism enables treating problems that arise in many application domains such as engineering [1], sociology [2] or biology [3]. Consensus and synchronization comes in different flavors: linear [4] or nonlinear [5] agent dynamics, fixed [6] or time-varying [4] interaction topology, continuous or discrete [7] time dynamics.

In most of the existing works, the control cost related to synchronization is not studied and the objective is related to the convergence of trajectories to a common one. In [8], the authors proposed an energy-aware control design that takes into account an overall cost and should reduce the communication and computation loads. In [9], the authors describe the problem of optimal control with a global cost in the multiagent framework. The problem is defined when the dynamics and the initial state for all agents are perfectly known and is NP-hard due to the information structure imposed by the

This work was partially supported by the ANR under the grant HANDY ANR-18-CE40-0010 and by INS2I CNRS under the 80'PRIME call.

The authors are with Université de Lorraine, CNRS, CRAN, F-54000 Nancy, France, \{jomphop.veetaseveera, vineeth.satheeskumar-varma, constantin.morarescu, jamal.daafouz\} @univ-lorraine.fr graph. Consequently, the authors design a distributed suboptimal controller. More recently, in [10], the authors present the design of a decentralized control strategy that allows singularly perturbed multi-agent systems to achieve synchronization with global performance guarantees. Additionally, [10] assumes that all agents utilize the same gain, which can be restrictive and even undesirable in some cases. In contrast, our objective is to guarantee that the cost incurred by each agent does not exceed a given bound during the process of synchronization. This can be of practical relevance in applications such as automatic cruise control on highways where each vehicle wants to follow the vehicle in front of it (common objective of synchronization), but also wants to ensure that its fuel consumption is not too high (individual cost). In such applications, considering a global cost might not be fair to each individual vehicle.

Since each agent has its own individual cost, standard optimization or optimal control approaches cannot be directly applied. In this context, we are inspired by notions in game theory, specifically that of satisfaction equilibrium and satisfaction games, introduced in [11]. A set of actions are said to be in satisfaction equilibrium when the individual cost for each agent is upper-bounded by a given threshold. This notion was applied to wireless networks in order to guarantee a satisfactory quality of service [12]. Note that we use game theory as an inspiration for some concepts and formalism, and not for the mathematical tools.

The rest of the paper is organized as follows. In Section II we describe the problem that we want to solve. In Section III we introduce the notion of satisfaction games and provide conditions to check if a set of gains are in satisfaction equilibrium. In Section IV, we provide a method to synthesize gains which are in satisfaction equilibrium for a general graph. Finally, in Section V we use some numerical examples to illustrate the results.

Notation: In the sequel, we use $(.)^{\top}$ for the transpose and $\otimes$ is the Kronecker product of two matrices. We denote by $\mathscr{N}(A)$ and $\mathscr{R}(A)$ the kernel and the image of the matrix $A$. The matrix $I_{n} \in \mathbb{R}^{n \times n}$ denotes the identity matrix and $\mathbf{1}_{n}$ denotes the column vector of ones having dimension $n$. We use $\operatorname{diag}\left(A_{1}, \ldots, A_{p}\right)$ to denote a block diagonal matrix having the blocks $A_{1}, \ldots, A_{p}$ on its diagonal. The element $e_{i} \in \mathbb{R}^{n}$ denotes the vector with a 1 in the $i^{\text {th }}$ coordinate and 0 elsewhere. The norm of a matrix $\|A\|$ is the largest singular values of $A$. Finally, $A^{\perp}$ denotes a matrix with the following properties : $\mathscr{N}\left(A^{\perp}\right)=\mathscr{R}(A)$ and $A^{\perp} A^{\perp \top}>0$. 


\section{PROBLEM FORMULATION}

\section{A. System dynamics}

We consider a network of $n \in \mathbb{N}$ agents, where the interactions are described by a graph $G=(V, E)$, with $V=\{1,2, \ldots, n\}$ the set of vertices and $E \subset V \times V$ the set of edges. We identify each agent with its index $i \in V$ and $\mathcal{N}_{i}=\{j \in V \mid(i, j) \in E\}$ specifies the neighborhood of agent $i$. We assign to each agent $i$ a state $x_{i}(t) \in \mathbb{R}^{n_{x}}$ with dynamics described by

$$
\left\{\begin{array}{l}
\dot{x}_{i}=A x_{i}+B u_{i} \\
y_{i}=\sum_{k \in \mathcal{N}_{i}}\left(x_{k}-x_{i}\right)
\end{array}\right.
$$

where $x_{i}, y_{i} \in \mathbb{R}^{n_{x}}, u_{i} \in \mathbb{R}^{n_{u}}, A \in \mathbb{R}^{n_{x} \times n_{x}}, B \in \mathbb{R}^{n_{x} \times n_{u}}$ and $i \in V$. Here, $u_{i}$ is the control applied by agent $i$ which depends on the output $y_{i}$. Let $x(t)=\left(x_{1}^{\top}(t), \ldots, x_{n}^{\top}(t)\right)^{\top} \in$ $\mathbb{R}^{n \cdot n_{x}}$ and $u(t)=\left(u_{1}^{\top}(t), \ldots, u_{n}^{\top}(t)\right)^{\top} \in \mathbb{R}^{n \cdot n_{u}}$ be respectively the global state of the network and the control at time $t \in \mathbb{R}$.

Remark 1. Our results do not require $A, B$ to be identical i.e. our results hold when $A_{i}, B_{i}$ describe the dynamics for agent $i$, but we use a common $A, B$ for ease of exposition during the change of variables in Section IV.

Throughout the paper we use graph Laplacian matrix $L \in$ $\mathbb{R}^{n \times n}$ whose components are defined as

$$
L_{i j}=\left\{\begin{array}{ll}
-1 & \text { if } i \neq j \text { and } j \in \mathcal{N}_{i} \\
\left|\mathcal{N}_{i}\right| & \text { if } i=j \\
0 & \text { otherwise, }
\end{array} \quad \forall i, j \in V .\right.
$$

\section{B. The cost}

The control goal is to asymptotically synchronize the set of agents i.e. to ensure that $\lim _{t \rightarrow+\infty}\left\|x_{j}(t)-x_{i}(t)\right\|=0$, $\forall i, j \in V$. To achieve this common goal, each agent has to provide a certain control effort that has to be limited in many real applications. Therefore, we consider a per-agent cost $J_{i}$ defined as follows

$$
J_{i}:=\int_{0}^{\infty} \sum_{j \in \mathcal{N}_{i}}\left\|x_{j}(t)-x_{i}(t)\right\|^{2}+u_{i}^{\top}(t) R_{i}^{\top} R_{i} u_{i}(t) \mathrm{d} t
$$

where the matrix $R_{i} \in \mathbb{R}^{n_{u} \times n_{u}}$ represents the weight given to the control action $u_{i}$.

Since we are looking for a decentralized control strategy, each agent only has access to the information on the relative state with respect to its neighborhood $\mathcal{N}_{i}$, i.e. $y_{i}(t)$ and does not have access to overall network state $x(t)$. Optimization of the cost $J_{i}$ cannot be achieved, unless $x(0)$ and $u_{j}$ are known for all $j \neq i$ (see [9] for details).

\section{GAME THEORETIC PRELIMINARIES}

In contrast with an optimal control approach that is adopted in [9], our problem involves a cost $J_{i}$ associated with each agent. Since we have each agent desiring to reduce its individual cost, we are in the framework of a game. The agents only have information from their neighbors and not from the whole network i.e. $u_{i}(t)$ must be designed independently of $x(0)$ and must depend only on $y_{i}(t)$. Besides the general information structure, the agents also use information from past actions and take this into account for their current and future actions. This case corresponds to a differential game with incomplete information [13]. However, the research on differential games remains open for a general information structure. Therefore, we are inspired by [11], which introduces the notion of satisfaction games. In a satisfaction game, when all players have

$$
J_{i} \leq \gamma\left\|x(0)-\mathbf{1}_{n} \otimes x_{i}(0)\right\|^{2}
$$

where $\gamma>0$ is a given threshold, the players are said to be in satisfaction equilibrium. In the following, we describe how we can model our problem as a satisfaction game, and in the subsequent section, we use results in LQ control with static output feedback to provide conditions on achieving a satisfaction equilibrium.

We are restricted to designing a controller $u_{i}$ based on the output $y_{i}$. In this setting, we search for controllers that are of static-output feedback type.

Assumption 1. The controller for agent $i$ is of the form $u_{i}=$ $K_{i} y_{i}$, where $K_{i} \in \mathscr{K}_{i}$ with $\mathscr{K}_{i}=\mathbb{R}^{n_{u} \times n_{x}}$.

With Assumption 1, the control strategy for agent $i$ is fully defined by the choice of gain $K_{i}$. Next, it is important to note that the cost $J_{i}$ will always depend on the global initial state $x(0)$. However, this dependency can be removed by treating the case in which all the other agents start in a ball of unit radius around each agent. The resulting cost can then be scaled up for other initial conditions.

Remark 2. To simplify the writing in the following we scale the initial condition such that $\left\|x(0)-\mathbf{1}_{n} \otimes x_{i}(0)\right\|^{2} \leq$ $1, \forall i \in V$. As usual the individual costs scales with the initial condition and under the normalization above we get a satisfaction game when $J_{i} \leq \gamma, \forall i \in V$.

We define a gain profile as $\mathbf{K}=\left(K_{1}, \ldots, K_{n}\right) \in \mathscr{K}$, where $\mathscr{K}=\mathscr{K}_{1} \times \ldots \times \mathscr{K}_{n}$. We also use $K_{-i}:=$ $\left(K_{1}, \ldots, K_{i-1}, K_{i+1}, \ldots, K_{n}\right)$ to denote the profile of gains for all agents except $i$. To emphasize its $i$-component, we write the vector $\mathbf{K}$ as $\left(K_{i}, K_{-i}\right)$ with a slight abuse of notation. Under Assumption 1 we define the satisfaction game as follows.

The satisfaction game in standard form is defined by the ordered triplet

$$
\mathcal{G}=\left(V,\left\{\mathscr{K}_{i}\right\}_{i \in V},\left\{f_{i}\right\}_{i \in V}\right),
$$

where

- $V=\{1, \ldots, n\}$ is the set of agents and we identify each agent $i$ as player $i$,

- $\mathscr{K}_{i}=\mathbb{R}^{m \times n_{x}}$ is the set of actions or gains $K_{i}$ applied by player $i$, and the control applied by each player is given according to Assumption 1,

- A player $i$ is said to be satisfied when his action $K_{i} \in$ $f_{i}\left(K_{-i}\right)$ considering the actions of the other players $K_{-i}$ 
are given. Here, $f_{i}\left(K_{-i}\right)$ is called as the satisfaction function, and is defined as follows

$$
f_{i}\left(K_{-i}\right):=\left\{K_{i} \in \mathscr{K}_{i} \mid J_{i}\left(K_{i}, K_{-i}\right)<\gamma\right\} .
$$

The satisfaction function $f_{i}$ of the player $i$ describes the set of actions which guarantees that his cost is upper-bounded by $\gamma$ given the actions of all the other players. This allows us to define the satisfaction equilibrium as follows.

Definition 1. Let $\mathcal{G}$ be a strategic game and $f_{1}, \ldots, f_{n}$ be $n$ set-valued satisfaction functions. The strategy profile $\mathbf{K}^{*}=$ $\left(K_{1}^{*}, \ldots, K_{n}^{*}\right)$ is a satisfaction equilibrium (SE) if and only if

$$
\forall i \in V, K_{i}^{*} \in f_{i}\left(K_{-i}^{*}\right)
$$

With these definitions, the purpose of each player is to be satisfied, so an equilibrium is when $J_{i}\left(\mathbf{K}^{*}\right) \leq \gamma$ for all $i \in V$. Once the players are at the SE, none of them have a particular interest to change their current actions as each player has achieved the desired bound on his cost. They are assumed to be careless of the satisfaction of other players.

\section{MAIN RESULTS}

\section{A. Change of variables}

In order to check if $\mathbf{K}^{*}$ is an SE, we reduce the synchronization problem to a stabilization problem by a change of variables on (1). For analysis purpose, for each $i \in V$, we introduce the transformed state vector:

$$
\chi_{i}=\left(x_{1}^{\top}-x_{i}^{\top}, \ldots, x_{i-1}^{\top}-x_{i}^{\top}, x_{i+1}^{\top}-x_{i}^{\top}, \ldots, x_{n}^{\top}-x_{i}^{\top}\right)^{\top}
$$

and $\chi_{i} \in \mathbb{R}^{(n-1) \times n_{x}}$. Denote by $\mathcal{L}_{-i}$, the Laplacian matrix $L$ without the $i$-th row and column, and by $\mathcal{L}_{i}$, the $i$-th row of $L$. Finally, we also use $\mathcal{L}_{i \text { :red }}$ to denote the row matrix $\mathcal{L}_{i}$ with the $i$-th column removed. We can now write the dynamics for $\chi_{i}$ as

$$
\dot{\chi}_{i}=\mathcal{A}_{i}\left(K_{-i}\right) \chi_{i}+\mathcal{B}_{i} u_{i}
$$

where

$$
\begin{gathered}
\mathcal{A}_{i}:=I_{n-1} \otimes A-\left(I_{n-1} \otimes B\right) \operatorname{diag}\left(K_{-i}\right)\left(\mathcal{L}_{-i} \otimes I_{n_{x}}\right), \\
\mathcal{B}_{i}=-I_{n-1} \otimes B .
\end{gathered}
$$

The term $\operatorname{diag}\left(K_{-i}\right)$ is not a control action but it represents the behaviour of the network. For agent $i$, the control action is simply $u_{i}=K_{i} y_{i}$ as it can not control the other agents. Let us define the auxiliary variables

$$
\left\{\begin{aligned}
z_{i} & =\mathcal{C}_{i} \chi_{i}+\mathcal{D}_{i} u_{i} \\
y_{i} & =\mathcal{F}_{i} \chi_{i} \\
u_{i} & =K_{i} y_{i}
\end{aligned}\right.
$$

where

$$
\mathcal{C}_{i}=\left(\begin{array}{c}
\operatorname{diag}\left(\mathcal{L}_{i \text { red }}\right) \otimes I_{n_{x}} \\
\mathbf{0}_{n_{u} \times(n-1) n_{x}}
\end{array}\right), \mathcal{D}_{i}=\left(\begin{array}{c}
\mathbf{0}_{(n-1) n_{x} \times n_{u}} \\
R_{i}
\end{array}\right),
$$

and $\mathcal{F}_{i}=-\mathcal{L}_{i \text { :red }} \otimes I_{n_{x}}$. Since the $n$-th block of $\mathcal{C}_{i}$ is 0 by definition, the $n$-th block of $z_{i}$ will contain the weighted control $R_{i} u_{i}$. The cost for any agent $i$ can now be written in terms of $z_{i}$ as

$$
J_{i}=\int_{0}^{\infty}\left\|z_{i}(t)\right\|^{2} \mathrm{~d} t
$$

Under the new variables, the problem of asymptotic synchronization of the system (1) now becomes stabilization of the system (4). This allows us to establish the following result.

Proposition 1. Let a gain profile $\mathbf{K}^{*}$ be given. The following statements are equivalent when Assumptions 1 holds.

1) The gain profile $\mathbf{K}^{*}$ is an SE of the satisfaction game $\mathcal{G}$ that stabilizes (4)-(5) $\forall i \in V$.

2) For all $i \in V$, there exists a positive-definite matrix $P_{i}>0$ such that

$$
\left\{\begin{array}{c}
P_{i} \mathcal{A}_{i, c l}\left(\mathbf{K}^{*}\right)+\mathcal{A}_{i, c l}\left(\mathbf{K}^{*}\right)^{\top} P_{i}+\mathcal{C}_{i, c l}^{\top} \mathcal{C}_{i, c l}<0 \\
P_{i}-\gamma I_{(n-1) n_{x}}<0
\end{array}\right.
$$

where $\mathcal{A}_{i, c l}\left(\mathbf{K}^{*}\right)=\left(\mathcal{A}_{i}\left(K_{-i}^{*}\right)+\mathcal{B}_{i} K_{i}^{*} \mathcal{F}_{i}\right), \mathcal{C}_{i, c l}=\left(\mathcal{C}_{i}+\right.$ $\left.\mathcal{D}_{i} K_{i}^{*} \mathcal{F}_{i}\right)$ are respectively the closed-loop matrices for $\chi_{i}$ and $z_{i}$.

Proof. Lemma 1 in [14] states that, when $K_{i}^{*}$ is given and $\left\|\chi_{i}(0)\right\| \leq 1$ is known, the following are equivalent:

- The gain $K_{i}^{*}$ stabilizes the system (4)-(5) and yields the LQ cost $J_{i}\left(K_{i}^{*}, K_{-i}^{*}\right)<\gamma$

- There exists $P_{i}>0$ such that

$$
P_{i} \mathcal{A}_{i, c l}\left(\mathbf{K}^{*}\right)+\mathcal{A}_{i, c l}\left(\mathbf{K}^{*}\right)^{\top} P_{i}+\mathcal{C}_{i, c l}^{\top} \mathcal{C}_{i, c l}<0
$$

and $\left\|P_{i}\right\|<\gamma$.

Proof of 1$) \Rightarrow 2$ ). Let $\mathbf{K}^{*}$ be an SE of $\mathcal{G}$ that stabilizes (4)(5) $\forall i \in V$. By Definition 1, one has $K_{i}^{*} \in f_{i}\left(K_{-i}^{*}\right), \forall i \in V$ yielding $J\left(K_{i}^{*}, K_{-i}^{*}\right)<\gamma, \forall i \in V$. We rewrite (4)-(5) in closed-loop form as

$$
\dot{\chi}_{i}=\mathcal{A}_{i, c l}\left(\mathbf{K}^{*}\right) \chi_{i}
$$

with the cost given by

$$
J_{i}=\int_{0}^{\infty}\left\|\mathcal{C}_{i, c l} \chi_{i}\right\|^{2} \mathrm{~d} t<\gamma,
$$

for all $i \in V$. Since $\mathbf{K}^{*}$ is given, the matrices $\mathcal{A}_{i, c l}\left(\mathbf{K}^{*}\right)$ are fixed and known. From Remark 2, we have $\left\|\chi_{i}(0)\right\| \leq 1$ and applying Lemma 1 in [14] one obtains the existence of matrices $P_{i}$ satisfying (7).

Proof of 2) $\Rightarrow 1$ ). Suppose now that (7) holds $\forall i \in V$. Again, from Lemma 1 in [14], one has that $K_{i}^{*}$ stabilizes (4)-(5) and $J\left(K_{i}^{*}, K_{-i}^{*}\right)<\gamma$ for all $i \in V$. Therefore, $K_{i}^{*} \in f_{i}\left(K_{-i}^{*}\right), \forall i \in V$ which means that $\mathbf{K}^{*}$ is an SE of $\mathcal{G}$ given a $\gamma$.

To summarize, we have provided LMIs conditions to test if a given $\mathbf{K}$ is an $\mathrm{SE}$ of the game $\mathcal{G}$ for the performance bound $\gamma$. The given $\mathbf{K}$ is a satisfaction equilibrium of $\mathcal{G}$ if and only if it satisfies 2) for all $i$. The inability to find matrices $P_{i}$ however does not imply that they do not exist, this may arise due to numerical issues with the LMI solver. Finally, for a given $\gamma$, $\mathbf{K}$ may not be unique and it is possible to have several gains which are satisfaction equilibria. Therefore, we define the set of satisfaction equilibria as

$$
\mathcal{K}^{*}=\left\{\mathbf{K}^{*}: \forall i \in V, K_{i}^{*} \in f_{i}\left(K_{-i}^{*}\right)\right\} .
$$


In the next subsection, we provide a method which allows us to synthesize the gains for a given thereshold $\gamma$.

\section{B. Synthesis of a $\mathbf{K} \in \mathcal{K}^{*}$}

In this section, we first present conditions that allow us to generate the satisfaction function $f_{i}\left(K_{-i}\right)$ based on the results in [14]. In the following proposition, for a given set of gains $K_{-i}$, we find a synchronizing gain $K_{i}$ under certain conditions as described below.

Proposition 2 (Based on Theorem 1 in [14]). Let the set of gains $K_{-i}$ be given. Consider the sets

$$
\begin{aligned}
& \mathscr{X}_{i}\left(K_{-i}\right):=\left\{X \in \mathbb{R}^{(n-1) \cdot n_{x} \times(n-1) \cdot n_{x}}:\right. \\
& \left.\left[\begin{array}{c}
\mathcal{B}_{i} \\
\mathcal{D}_{i}
\end{array}\right]^{\perp}\left[\begin{array}{cc}
\mathcal{A}_{i} X+X \mathcal{A}_{i}^{\top} & X \mathcal{C}_{i}^{\top} \\
\mathcal{C}_{i} X & -I_{(n-1) . n_{x}}
\end{array}\right]\left[\begin{array}{c}
\mathcal{B}_{i} \\
\mathcal{D}_{i}
\end{array}\right]^{\perp \top}<0\right\}, \\
& \mathscr{Y}_{i}\left(K_{-i}\right):=\left\{Y \in \mathbb{R}^{(n-1) \cdot n_{x} \times(n-1) \cdot n_{x}}:\right. \\
& \mathcal{F}_{i}^{\top \perp}\left(Y \mathcal{A}_{i}+\mathcal{A}_{i}^{\top} Y+\mathcal{C}_{i}^{\top} \mathcal{C}_{i}\right) \mathcal{F}_{i}^{\top \perp \top}<0, \\
& \left.Y-\gamma I_{(n-1) . n_{x}}<0\right\} \text {. }
\end{aligned}
$$

Under Assumption 1, if no $P>0$ exist such that $P^{-1} \in$ $\mathscr{X}_{i}\left(K_{-i}\right)$ and $P \in \mathscr{Y}_{i}\left(K_{-i}\right)$, then $f_{i}\left(K_{-i}\right)=\emptyset$, which implies that we can not find a suitable $K_{i}$ such that $\left(K_{i}, K_{-i}\right)$ is a satisfaction equilibrium. Otherwise, the satisfaction function for the game $\mathcal{G}$ is given by

$$
\begin{array}{r}
f_{i}\left(K_{-i}\right)=\left\{-\rho_{i} \mathscr{B}_{i}^{\top} \Phi_{i}\left(K_{-i}\right) \mathscr{C}_{i}^{\top}\left(\mathscr{C}_{i} \Phi_{i}\left(K_{-i}\right) \mathscr{C}_{i}^{\top}\right)^{-1}+\right. \\
\rho_{i} S_{i}\left(K_{-i}\right)^{1 / 2} M_{i}\left(\mathscr{C}_{i} \Phi\left(K_{-i}\right) \mathscr{C}_{i}^{\top}\right)^{-1 / 2}: P>0, \\
\left.P^{-1} \in \mathscr{X}\left(K_{-i}\right), P \in \mathscr{Y}\left(K_{-i}\right),\|M\|<1\right\},
\end{array}
$$

where

$$
\begin{aligned}
& \mathscr{B}_{i}:=\left[\begin{array}{c}
P \mathcal{B}_{i} \\
\mathcal{D}_{i}
\end{array}\right], \quad \mathscr{C}_{i}:=\left[\begin{array}{ll}
\mathcal{F}_{i} & 0
\end{array}\right], \\
& Q_{i}\left(K_{-i}\right):=\left[\begin{array}{cc}
P \mathcal{A}_{i}\left(K_{-i}\right)+\mathcal{A}_{i}\left(K_{-i}\right)^{\top} P & \mathcal{C}_{i}^{\top} \\
\mathcal{C}_{i} & -I_{(n-1) . n_{x}}
\end{array}\right], \\
& \rho_{i}\left(K_{-i}\right):=\rho_{\min }\left(K_{-i}\right)+p, \\
& \rho_{i, \min }\left(K_{-i}\right):=\max \left\{0, \lambda_{\max }\left[\mathscr { B } _ { i } ^ { + } \left(Q_{i}\left(K_{-i}\right)-\right.\right.\right. \\
& \left.\left.\left.Q_{i}\left(K_{-i}\right) \mathscr{B}_{i}^{\perp \top}\left(\mathscr{B}_{i}^{\perp} Q_{i}\left(K_{-i}\right) \mathscr{B}_{i}^{\perp \top}\right)^{-1} \mathscr{B}_{i}^{\perp} Q_{i}\left(K_{-i}\right)\right) \mathscr{B}_{i}^{+\top}\right]\right\}, \\
& \Phi_{i}\left(K_{-i}\right):=\left(\rho_{i} \mathscr{B}_{i} \mathscr{B}_{i}^{\top}-Q_{i}\left(K_{-i}\right)\right)^{-1}, \\
& S_{i}\left(K_{-i}\right)=\rho_{i} I_{n_{x}}-\mathscr{B}_{i}^{\top}\left[\Phi_{i}-\Phi_{i} \mathscr{C}^{\top}\left(\mathscr{C}_{i} \Phi_{i} \mathscr{C}_{i}^{\top}\right)^{-1} \mathscr{C}_{i} \Phi_{i}\right] \mathscr{B}_{i},
\end{aligned}
$$

with $p \in \mathbb{R}_{\geq 0}$ an arbitrary non-negative scalar.

Proof. By definition of the satisfaction function, we have $f_{i}\left(K_{-i}\right)=\left\{K_{i} \mid J_{i}\left(K_{i}, K_{-i}\right)<\gamma\right\}$. Therefore, for a given $K_{-i}, f_{i}$ is the set of gains which result in a cost bounded by $\gamma$. Theorem 1 in [14] provides conditions on the existence of a stabilizing static output feedback gain such that the LQ cost is bounded by a given factor $\gamma$, when $\chi_{i}(0)=W w_{0}$ with $\left\|w_{0}\right\|=1$. From Remark 2 , we have that $\left\|\chi_{i}(0)\right\| \leq 1$. Once we rewrite the synchronization problem as (4) and (5), for a given $K_{-i}$, finding $K_{i}$ which results in $J_{i}\left(K_{i}, K_{-i}\right)<\gamma$ is transformed into a problem of static output feedback with a bounded LQ cost. Since we take $\left\|W w_{0}\right\| \leq 1$, the condition $W^{\top} Y W-\gamma I<0$ as required in [14] is satisfied if $Y-\gamma I_{(n-1) n_{x}}<0$. Applying the theorem, we get that the following are equivalent:

- There exists $K_{i}$ stabilizing (4)-(5) such that $J_{i}\left(K_{i}, K_{-i}\right)<\gamma$
- There exists $P>0$ such that $P^{-1} \in \mathscr{X}\left(K_{-i}\right)$ and $P \in \mathscr{Y}\left(K_{-i}\right)$ and $K_{i}$ is given by $\quad-\rho \mathscr{B}^{\top} \Phi\left(K_{-i}\right) \mathscr{C}^{\top}\left(\mathscr{C} \Phi\left(K_{-i}\right) \mathscr{C}^{\top}\right)^{-1} \quad+$ $\rho S\left(K_{-i}\right)^{1 / 2} M\left(\mathscr{C} \Phi\left(K_{-i}\right) \mathscr{C}^{\top}\right)^{-1 / 2}$.

Therefore, if no such $P$ exists, the satisfaction function is the empty-set. Otherwise, it can be written as in (10).

Section 4 of [14] provides a scaled min-max algorithm which generates a matrix $P>0$ satisfying the conditions in Proposition 2, when the problem has a solution. It is noteworthy that our design remains centralized although its implementation is distributed. Indeed, in order to find a $\mathbf{K}$ which satisfies Proposition 1, we need to find the gains $K_{i}$ for all agents. To do that, we propose an algorithm which we call the Sequential Satisfaction Response (SSR) in order to achieve this task. This algorithm is inspired by the sequential best response algorithm that is commonly found in the literature on game theory [13]. In contrast with the classical best response algorithm where the actions of players must converge over iterations, our algorithm only needs to satisfy $\mathbf{K} \in \mathcal{K}^{*}$ to have succeeded.

Data: Initialize $\mathbf{K}$ with synchronizing gains;

Data: $\mathrm{i}=1 ; \mathrm{j}=0$;

while $\mathbf{K} \notin \mathcal{K}^{*}$ (verified using Proposition 1) do

if $P>0$ exists satisfying Proposition 2 given $K_{-i}$ then

Update $K_{i}$ using (10) and a feasible $P$; $\mathrm{j}=0$;

else $\mathrm{j}=\mathrm{j}+1$; if $j \geq n$ then | Result: Stop algorithm, failure to find SE. end

end

$i=(i \bmod n)+1$

end

Result: Found an equilibrium.

Algorithm 1: Sequential satisfaction response

Algorithm 1 applies the scaled min-max algorithm in [14] repeatedly to find $P>0$ satisfying the conditions in Proposition 2 given $K_{-i}$. This results in a gain for player $i$ which satisfies his cost requirement. The player index is then updated to the next player and this procedure is repeated until $\mathbf{K}$ is a satisfaction equilibrium. Even if the algorithm fails to find a gain for a player $i$, it does not immediately stop and the player index is updated. However, if all $n$ players consecutively fail to find a gain $K_{i}$, the algorithm is stopped. Indeed, it is possible that the algorithm never stops as only a certain number of players are satisfied with this set cycling or not changing. We have no theoretical guarantee that this algorithm will find a satisfaction equilibrium even if $\left|\mathcal{K}^{*}\right|>0$. In case of the algorithm failing, a larger $\gamma$ may be considered in order to find a satisfaction equilibrium for the game with the larger $\gamma$. This equilibrium may then be used as input for initializing the algorithm with the smaller $\gamma$. Future works will explore improving the algorithm by adapting $\gamma$, but theoretical results 
are hard to obtain due to the conditions for finding the gains for just one player being non-convex and requiring the minmax algorithm. This difficulty is inherited from the problem of static output feedback design for linear systems and is not related to the multi-agent systems.

If all agents have the same structure of $y_{i}$ and the dynamics are identical even if the indices are permuted the computation load is reduced by a factor of $\mathrm{n}$. This type of graphs is called in the literature (see [15]) prime w.r.t. the modular decomposition. This means that the agents react the same way, so it allows us to look for a symmetric satisfaction equilibrium, i.e. we look for $K_{0}$ such that $\mathbf{1}_{n}^{\top} \otimes K_{0} \in \mathcal{K}$. For a given $K_{0}$, we can apply Proposition 1 to get that $\mathbf{1}_{n}^{\top} \otimes K_{0} \in \mathcal{K}^{*}$ if and only if there exists $P>0$ such that

$$
\begin{gathered}
P \mathcal{A}_{i, c l}\left(\mathbf{1}_{n}^{\top} \otimes K_{0}\right)+\mathcal{A}_{i, c l}\left(\mathbf{1}_{n}^{\top} \otimes K_{0}\right)^{\top} P \\
+\mathcal{C}_{i, c l}^{\top} \mathcal{C}_{i, c l}<0, \quad\|P\|<\gamma .
\end{gathered}
$$

for any $i \in V$. Due to symmetry, we have $A_{i, c l}=A_{j, c l}$ and $C_{i, c l}=C_{j, c l}$ for any $i, j \in V$. Therefore instead of having to solve $n$ LMIs, we just need to solve one. We can also simplify the synthesis with the following result.

Corollary 1. Let the network be prime w.r.t. the modular decomposition. Under Assumptions 1, an SE for dynamics (1) with individual costs (2) is given by $\mathbf{1}_{n}^{\top} \otimes K_{0}$ if and only if there exists $P>0,\|M\|<1$ such that

$$
\begin{aligned}
& P^{-1} \in \mathscr{X}_{i}\left(\mathbf{1}_{n-1}^{\top} \otimes K_{0}\right), P \in \mathscr{Y}_{i}\left(\mathbf{1}_{n-1}^{\top} \otimes K_{0}\right) \\
& K_{0}=-\rho_{i} \mathscr{B}_{i}^{\top} \Phi_{i}\left(\mathbf{1}_{n-1}^{\top} \otimes K_{0}\right) \mathscr{C}_{i}^{\top}\left(\mathscr{C}_{i} \Phi_{i}\left(\mathbf{1}_{n-1}^{\top} \otimes K_{0}\right) \mathscr{C}_{i}^{\top}\right)^{-1} \\
& +\rho_{i} S_{i}\left(\mathbf{1}_{n-1}^{\top} \otimes K_{0}\right)^{1 / 2} M\left(\mathscr{C}_{i} \Phi_{i}\left(\mathbf{1}_{n-1}^{\top} \otimes K_{0}\right) \mathscr{C}_{i}^{\top}\right)^{-1 / 2}
\end{aligned}
$$

with $\mathscr{X}_{i}, \mathscr{Y}_{i}, \mathscr{C}_{i}, \Phi_{i}, S_{i}, \rho$ as defined in Proposition $2 \forall i \in V$.

Proof. The above result is obtained by directly applying Proposition 2 and by exploiting the fact that $K_{i}=K_{0}$ and $K_{-i}=\mathbf{1}_{n-1}^{\top} \otimes K_{0}$. Additionally, the closed-loop dynamics are identical for all $i \in V$.

Corollary 1 implies that for $K_{0}$ can be found as a solution to a fixed point equation. Algorithm 1 can be used in a simpler manner to find a suitable $K_{0}$ by fixing all agents outside an arbitrary agent $i$ to have some gain $K_{0}$ and then finding $K_{i}$ using Proposition 2. Then $K_{0}$ is updated to the $K_{i}$ and the process is repeated until $\mathbf{1}_{n}^{\top} \otimes K_{0} \in \mathcal{K}^{*}$. The main motivation for considering this special case is that the number of iterations does not scale with $n$ as it does in the general case. For large scale networks, obtaining the gains via the general algorithm may be computationally infeasible as both the system matrix dimensions and the iterations scale with $n$.

\section{NUMERICAL ILLUSTRATIONS}

In this section, we provide some numerical examples to illustrate the effectiveness of the algorithm proposed in this manuscript. Without any loss of generality, we consider only the following simple agent dynamics (1) with $A=1, B=1$ and $R_{i}=1$ for all $i$. We use $K_{i}^{*}$ and $J_{i}^{*}$ for the control gains and the corresponding costs obtained using the proposed strategy. On the other hand, $K_{i}^{o}$ and $J_{i}^{o}$ are the control gains and the corresponding costs obtained using the strategy in [10]. For both graphs, we provide the a posteriori values of cost functions obtained by implementing the controller gains profile $\mathbf{K}$. The costs are computed by using the initial condition $x_{0}$ and the state of the network. Through the second graph, we show that the constraints are still satisfied independently of the initial conditions.

\section{A. Ring directed graph}

Let us first consider a simple ring directed graph with 5 agents described by the following Laplacian matrix:

$$
L=\left(\begin{array}{ccccc}
1 & -1 & 0 & 0 & 0 \\
0 & 1 & -1 & 0 & 0 \\
0 & 0 & 1 & -1 & 0 \\
0 & 0 & 0 & 1 & -1 \\
-1 & 0 & 0 & 0 & 1
\end{array}\right)
$$

For the simulation, we use $\gamma=1.3$ and the results of the proposed algorithm are summarized in the table below.

\begin{tabular}{|c|c|c|c|c|c|}
\hline & 1 & 2 & 3 & 4 & 5 \\
\hline$x_{i}(0)$ & 0.67 & 0.67 & 0.31 & 0.01 & 0.02 \\
\hline$K_{i}^{*}$ & 3.54 & 3.54 & 3.52 & 3.51 & 3.60 \\
$J_{i}^{*}$ & 0.46 & 0.64 & 0.33 & 0.51 & 0.95 \\
\hline
\end{tabular}

We note that, due to the regularity of the graph the five gains are quite similar and the costs are bounded by $\gamma$. The synchronizing trajectories of the 5 agents are plotted in Fig. 1 and the corresponding control inputs are in Fig. 2.

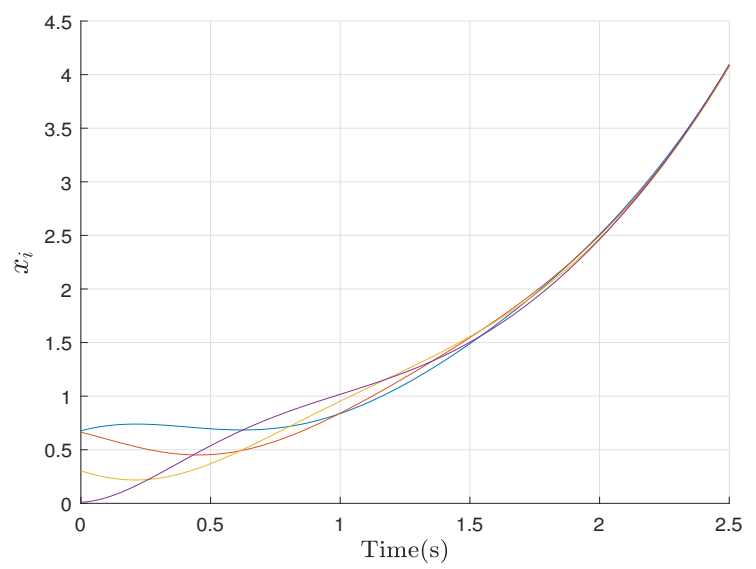

Fig. 1. Trajectories of the agents for the ring directed graph given by (13).

\section{B. Undirected graph: comparison with [10]}

In the following we consider an undirected graph with 8 agents in order to compare our design strategy with the one proposed in [10]. It is noteworthy that [10] proposes a decentralized control achieving synchronization with global performance guarantees for singularly perturbed multi-agents systems interacting over networks reprezented by undirected fixed graphs. It is imposed that all agents applies the same control gain and the synchronizing control design is reduced to a stabilizing control design for an uncertain system with bounded uncertainties. These uncertainties are related to the bounds on the maximum and minimum (non-zero) eigenvalues of the Laplacian. Solving a Riccati equation, one finds a 


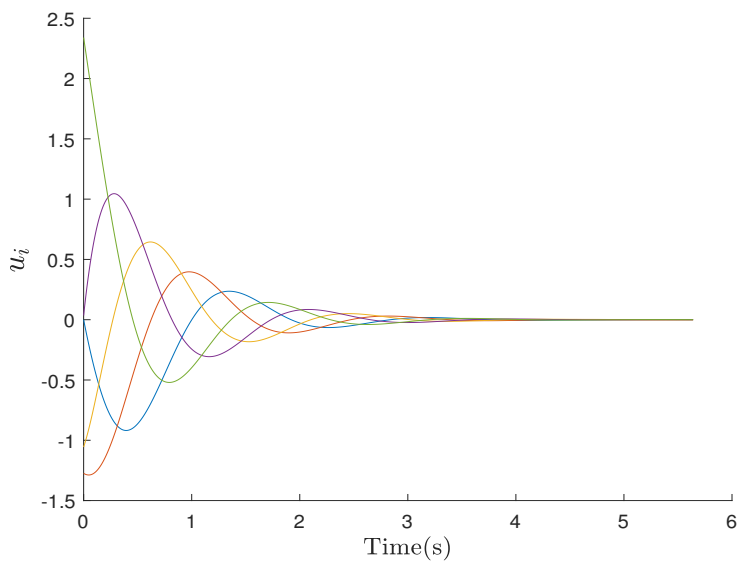

Fig. 2. Controls $u_{i}$ for the ring directed graph given by (13).

common gain for all agents ensuring that the global cost is upper-bounded. In order to highlight the improvements that we can obtain by using the approach proposed, in this simulation, we consider a graph in which the agent centralities are very different. For the example above, the controller gains are almost similar meaning that the strategy proposed in [10] might provide good results. However if the undirected graph $G$ is associated to the following Laplacian

$$
L=\left(\begin{array}{cccccccc}
7 & -1 & -1 & -1 & -1 & -1 & -1 & -1 \\
-1 & 2 & 0 & 0 & 0 & 0 & 0 & -1 \\
-1 & 0 & 2 & 0 & 0 & 0 & 0 & -1 \\
-1 & 0 & 0 & 2 & 0 & 0 & 0 & -1 \\
-1 & 0 & 0 & 0 & 2 & 0 & 0 & -1 \\
-1 & 0 & 0 & 0 & 0 & 2 & 0 & -1 \\
-1 & 0 & 0 & 0 & 0 & 0 & 2 & -1 \\
-1 & -1 & -1 & -1 & -1 & -1 & -1 & 7
\end{array}\right)
$$

one has no reason to apply the same controller gain to all the agents. For the simulation, we use $\gamma=0.6$ and the results of both strategies are summarized in the table below. We test 3 different initial conditions by varying $x_{0}$. We use $K^{*}=(0.39,1.30,1.30,1.30,1.30,1.30,1.30,0.39)$ found using Algorithm 1 , and $K^{\circ}=$ $(3.09,3.09,3.09,3.09,3.09,3.09,3.09,3.09)$ based on the results in [10].

\begin{tabular}{|l|l|l|l|l|l|l|l|l|l|}
\hline \multicolumn{2}{|c|}{$\gamma=0.6$} & 1 & 2 & 3 & 4 & 5 & 6 & 7 & 8 \\
\hline Case 1 & $x_{i}(0)$ & 0.50 & 0.1 & 0.4 & 0.1 & 0.4 & 0.4 & 0.1 & 0.04 \\
\hline & $J_{i}^{*}$ & 0.41 & 0.13 & 0.10 & 0.13 & 0.10 & 0.10 & 0.13 & 0.35 \\
& $J_{i}^{o}$ & 1.17 & 0.14 & 0.11 & 0.14 & 0.11 & 0.11 & 0.14 & 0.88 \\
\hline Case 2 & $x_{i}(0)$ & 0.04 & 0.53 & 0.04 & 0.46 & 0.04 & 0.46 & 0.04 & 0.53 \\
\hline & $J_{i}^{*}$ & 0.35 & 0.21 & 0.18 & 0.12 & 0.18 & 0.12 & 0.18 & 0.40 \\
& $J_{i}^{o}$ & 0.80 & 0.27 & 0.21 & 0.15 & 0.21 & 0.15 & 0.21 & 1.05 \\
\hline Case 3 & $x_{i}(0)$ & 0.44 & 0.47 & 0.03 & 0.07 & 0.02 & 0.54 & 0.06 & 0.54 \\
\hline & $J_{i}^{*}$ & 0.16 & 0.05 & 0.33 & 0.26 & 0.35 & 0.10 & 0.28 & 0.29 \\
& $J_{i}^{o}$ & 0.45 & 0.15 & 0.34 & 0.26 & 0.36 & 0.26 & 0.28 & 1.10 \\
\hline \multicolumn{7}{|c|}{ TABLE I } \\
\hline \multicolumn{7}{|c|}{}
\end{tabular}

UNDIRECTED GRAPH : INDIVIDUAL COST $J_{i}^{*}$ FOR DIFFERENT INITIAL CONDITIONS, $\gamma=0.6$

In Table I, $J_{i}^{*}$ and $J_{i}^{o}$ are respectively the costs incurred by agent $i$ using our strategy and the one in [10]. Since [10] bounds a global cost, we can compare their global cost to a total cost evaluated as $n \gamma$. The guaranteed overall bounds are $n \gamma=4.8$, for our strategy and 10.8 for the one in [10]. As seen from the table, each of individual costs are bounded by $\gamma$ for several possible initial conditions.

\section{CONCLUSION}

We study the problem of static-output feedback synchronization in a multi-agent system, which guarantees individual performance bounds. This problem is modeled as a satisfaction game and we seek gains that are in satisfaction equilibrium, i.e. the cost associated to each agent is upperbounded by a given $\gamma$. In this context, we provide conditions in the form of LMIs which can verify if a given set of gains are in satisfaction equilibrium. We provide a method to generate the gain for a certain agent when the gains for the other agents are known and this is used in an iterative algorithm which can synthesize a satisfaction equilibrium. Numerical examples illustrate our algorithm and compare our results with a previous result found in the literature.

\section{REFERENCES}

[1] F. Bullo, J. Cortes, and S. Martinez, Distributed control of robotic networks: a mathematical approach to motion coordination algorithms. Princeton University Press, 2009, vol. 27.

[2] R. Hegselmann, U. Krause et al., "Opinion dynamics and bounded confidence models, analysis, and simulation," Journal of artificial societies and social simulation, vol. 5, no. 3, 2002.

[3] G. A. Pavlopoulos, M. Secrier, C. N. Moschopoulos, T. G. Soldatos, S. Kossida, J. Aerts, R. Schneider, and P. G. Bagos, "Using graph theory to analyze biological networks," BioData mining, vol. 4, no. 1, p. 10 , 2011.

[4] A. Jadbabaie, J. Lin, and A. S. Morse, "Coordination of groups of mobile autonomous agents using nearest neighbor rules," IEEE Transactions on automatic control, vol. 48, no. 6, pp. 988-1001, 2003.

[5] I.-C. Morărescu, W. Michiels, and M. Jungers, "Effect of a distributed delay on relative stability of diffusely coupled systems, with application to synchronized equilibria," International Journal of Robust and Nonlinear Control, vol. 26, no. 7, pp. 1565-1582, 2016.

[6] R. Olfati-Saber and R. Murray, "Consensus problems in networks of agents with switching topology and time-delays," IEEE Transactions on Automatic Control, vol. 49, pp. 1520-1533, 2004.

[7] W. Ren and R. W. Beard, "Consensus seeking in multiagent systems under dynamically changing interaction topologies," IEEE Trans. on Automatic Control, vol. 50, no. 5, pp. 655-661, 2005.

[8] J. Hassan and J. Shamma, "Decentralized energy aware co-optimization of mobility and communication in multiagent systems," in 55th IEEE $C D C, 2016$.

[9] F. Borrelli and T. Keviczky, "Distributed lqr design for identical dynamically decoupled systems," IEEE Transactions on Automatic Control, vol. 53, no. 8, pp. 1901-1912, 2008.

[10] J. B. Rejeb, I.-C. Morărescu, and J. Daafouz, "Control design with guaranteed cost for synchronization in networks of linear singularly perturbed systems," Automatica, vol. 91, pp. 89-97, 2018.

[11] S. Ross and B. Chaib-draa, "Satisfaction equilibrium: Achieving cooperation in incomplete information games," in Conference of the Canadian Society for Computational Studies of Intelligence. Springer, 2006, pp. $61-72$.

[12] S. M. Perlaza, H. Tembine, S. Lasaulce, and M. Debbah, "Quality-ofservice provisioning in decentralized networks: A satisfaction equilibrium approach," IEEE Journal of Selected Topics in Signal Processing, vol. 6, no. 2, pp. 104-116, 2012.

[13] S. Lasaulce and H. Tembine, Game theory and learning for wireless networks: fundamentals and applications. Academic Press, 2011.

[14] T. Iwasaki, R. Skelton, and J. Geromel, "Linear quadratic suboptimal control with static output feedback," Systems \& Control Letters, vol. 23 , no. 6, pp. 421-430, 1994

[15] A. Brandstadt, V. B. Le, and J. Spinrad, Graph Classes: A Survey. SIAM Monographs on Discrete Mathematics and Applications, 1999. 